\title{
Dural metastasis of nasopharyngeal carcinoma: rare, but worth considering
}

\author{
Chin-Lung $\mathrm{KuO}^{1,3,4,5}$, MD, Donald Ming-Tak $\underline{\mathrm{Ho}}^{2,3,6}$, MD, FCAP, Ching-Yin $\underline{\mathrm{Ho}}^{1,3}$, MD, PhD
}

\begin{abstract}
Metastasis of nasopharyngeal carcinoma (NPC) to the dura, an extremely rare condition, can be symptomatically silent and mistaken for a benign entity radiographically. Missed diagnosis can lead to serious consequences or prove immediately fatal. We report a woman with dural metastasis of NPC that mimicked a meningioma on radiography. Craniectomy with tumour resection was performed due to rapid progression from the onset of symptoms to disability. The patient was still alive two years after surgery. This case emphasises the need to keep in mind the possibility of dural metastasis of NPC in patients with abnormal imaging features. This would not only avoid wrong and optimistic diagnosis, but also allow for appropriate treatment in a timely manner. To our knowledge, this is the first report of metastasis of NPC to the dura. We provide detailed information on the neoplastic lesion, which masqueraded as a benign entity and caused potentially fatal consequences.
\end{abstract}

Keywords: carcinoma, dura mater, meningioma, metastasis, nasopharyngeal

\section{INTRODUCTION}

Nasopharyngeal carcinoma (NPC), a malignancy highly prevalent in Southeast Asia, ${ }^{(1,2)}$ tends to develop distant metastases. The most common sites of distant metastasis involved are the bones, lungs and liver. ${ }^{(3)}$ Dural metastasis of NPC is extremely rare and can be symptomatically silent. It can also be mistaken for meningiomas radiographically due to identical dural tail enhancement on magnetic resonance (MR) imaging. ${ }^{(4)}$ This diagnostic dilemma may lead to mistaken identity, which can entail serious consequences or immediate death for the patient. In this study, we report a woman with dural metastasis of NPC that radiographically mimicked a meningioma.

\section{CASE REPORT}

A 37-year-old woman was diagnosed with differentiated nonkeratinising carcinoma of the nasopharynx (World Health Organization type IIA, T4N3aM0, 2002 American Joint Committee on Cancer stage IVB) in March 2009. Pretreatment MR imaging of the head and neck showed the presence of an invasive and extensive tumour involving bilateral aspects of the nasopharynx, left aspect of the oropharynx, parapharyngeal space, prevertebral muscle, skull base and cavernous sinus. Metastatic lymphadenopathy on both sides of the neck at the level II, III and IV lymph node regions and the left side of the neck at the level $\mathrm{V}$ lymph node region were also seen. There was confluence of the enlarged lymph nodes on the left side of the neck, which was over $6 \mathrm{~cm}$ in size. Two cycles of neoadjuvant chemotherapy with cisplatin, 5-fluorouracil and leucovorin (PFL) were given, followed by intensity-modulated radiotherapy (IMRT) concurrent with PFL. The total radiation dose was 70 Gy to the primary tumour and positive neck metastasis, and 60 Gy to the nasopharynx, oropharynx and bilateral neck levels II-V. Three more cycles of adjuvant chemotherapy with PFL were administered, and the full course of treatment was completed in September 2009.

In October 2009, follow-up MR imaging showed regression of the primary tumour and lymphadenopathy. However, an enhancing lesion, which was not noted during pretreatment MR imaging in March 2009, was observed in the left frontotemporal lobe with a dural tail sign (Fig. 1), giving rise to the suspicion of a meningioma. There was no recognised link between the nasopharynx and the lesion. As the possibility of tumour growth could not be excluded, we suggested that the patient be admitted for further evaluation and subsequent discussion at the head and neck oncology multidisciplinary team meeting. However, the patient refused admission due to personal reasons and chose observation. The patient remained well until November 2009, when she began suffering from intermittent episodes of confusion and difficulty in following complex commands. Nausea, vomiting and headaches developed at increasingly closer intervals, which steadily progressed to such an extent that she was unable to perform daily activities. Repeat MR imaging delineated a large space-occupying lesion in the left frontotemporal lobe, resulting in marked brain oedema (Fig. 2). However, no recurrent or residual tumour was found at the primary site.

Immediate craniectomy with tumour resection was performed for the patient. Histopathological analysis of the tumour revealed differentiated non-keratinising carcinoma (Fig. 3a) that had invaded the dura mater while sparing the

\footnotetext{
${ }^{1}$ Department of Otorhinolaryngology-Head and Neck Surgery, ${ }^{2}$ Department of Pathology and Laboratory Medicine, Taipei Veterans General Hospital, ${ }^{3}$ Department of Otorhinolaryngology, National Yang-Ming University School of Medicine, ${ }^{4}$ Department of Otorhinolaryngology, Taoyuan Armed Forces General Hospital, ${ }^{5}$ Institute of Brain Science, National Yang-Ming University, ${ }^{6}$ Department of Pathology and Laboratory Medicine, National Yang-Ming University of Medicine, Taipei, Taiwan

Correspondence: Dr Ching-Yin Ho, Associate Professor, Department of Otorhinolaryngology-Head and Neck Surgery, Taipei Veterans General Hospital, 201, Section 2, Shih-Pai Road, Taipei 112, Taiwan, ROC. cyho@vghtpe.gov.tw
} 

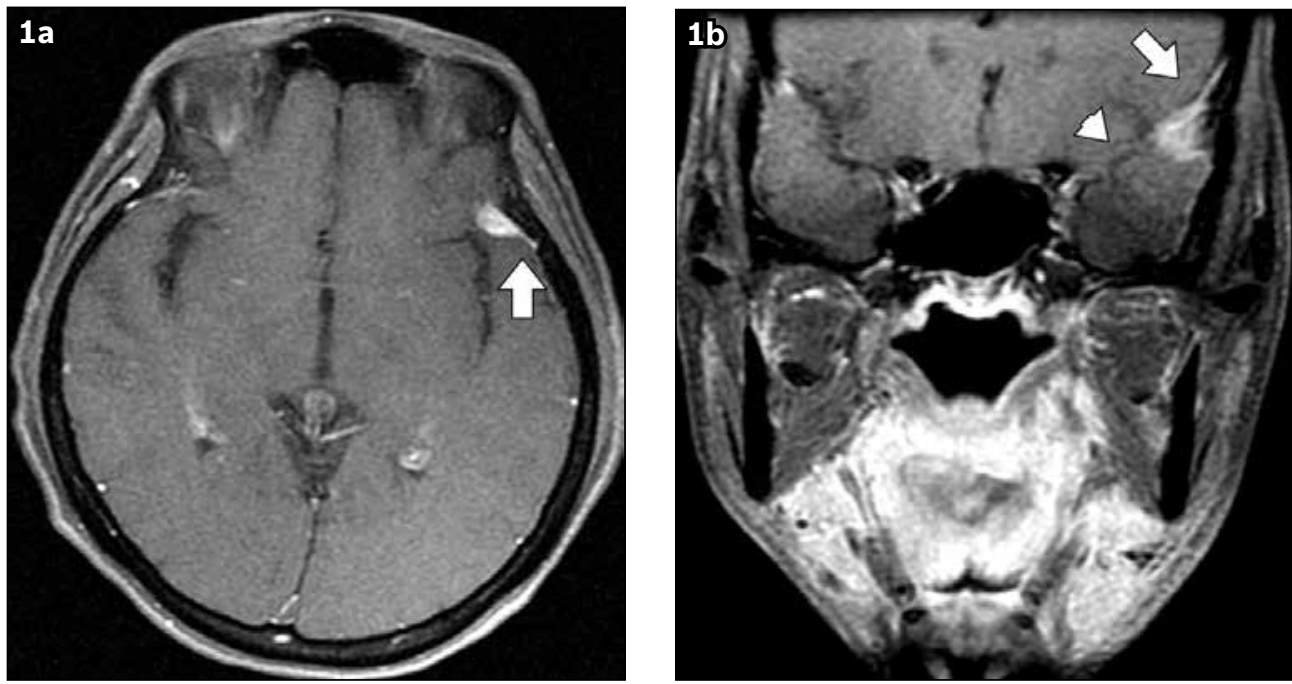

Fig. 1 Contrast-enhanced T1-weighted (a) axial and (b) coronal MR images one month after full course of treatment show a small heterogeneous enhancing lesion (arrows) in the left frontotemporal lobe with the dura tail sign in the vicinity of the lesion. No evident linkage was seen between the nasopharynx and the lesion (arrowhead).
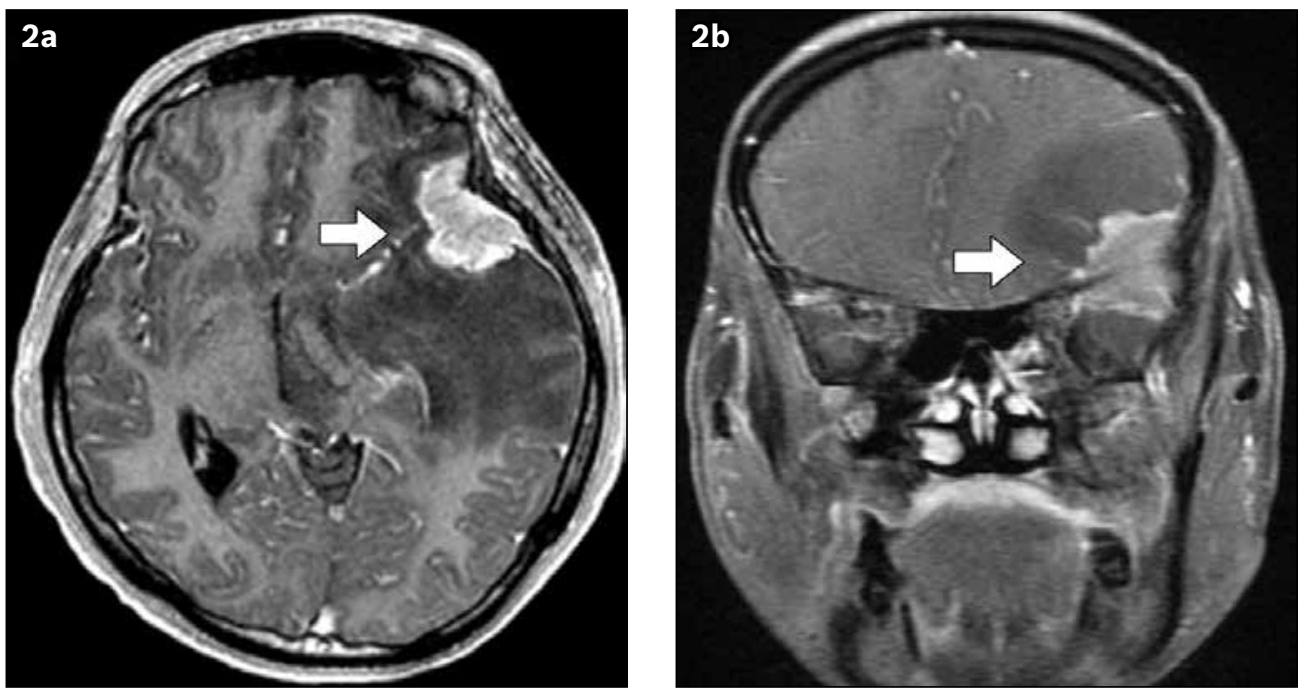

Fig. 2 Repeat MR images in (a) axial and (b) coronal views show rapid disease progression from a small lesion to an extensive lesion (arrows) with marked perifocal oedema, compression of the left lateral ventricle and an evident midline shift to the right side.

leptomeninges and brain parenchyma. The tumour type was consistent with the original nasopharyngeal biopsy taken in March 2009. In situ hybridisation performed for Epstein-Barr virus-encoded RNA was positive (Fig. 3b). The results of histopathological examinations implied dural metastasis from NPC. Two cycles of palliative chemotherapy with paclitaxel and ifosfamide concurrent with radiotherapy to the surgical bed were consequently given to alleviate the physical symptoms and to slow the progress of cancer enough to extend the patient's life. She was still alive two years following surgery.

\section{DISCUSSION}

Compared with Western countries, NPC is endemic in Southeast Asia and hyperendemic in Taiwan, in particular. ${ }^{(1,2)}$ The current standard treatment for advanced NPC is chemotherapy in addition to definitive radiotherapy to decrease distant metastasis and improve locoregional control, as well as for improved overall survival. ${ }^{(5)}$ Chemotherapy is administered concurrently and sequentially, either as induction therapy prior to definitive radiotherapy or as adjuvant therapy following definitive radiotherapy.(5) At Taipei Veterans General Hospital, Taiwan, IMRT has been adopted as an advanced mode of high-precision radiotherapy that can direct a high dose of radiation to the tumour bed while sparing normal tissue. The tumour control rate may be improved by using this technique.

Although significant advances have been made in the integration of radiation therapy and chemotherapy for the treatment of patients with NPC, distant metastasis remains a major cause of mortality and treatment failure in these patients. NPC has a propensity to develop distant metastases, with an incidence of $17 \%-53 \%$, and its most common metastatic sites are the bones, lungs and liver. ${ }^{(3)}$ Intracranial metastases, found in $25 \%$ of patients with malignancies, may be categorised by location as skull, dural, leptomeningeal and brain metastases. ${ }^{(6)}$ 

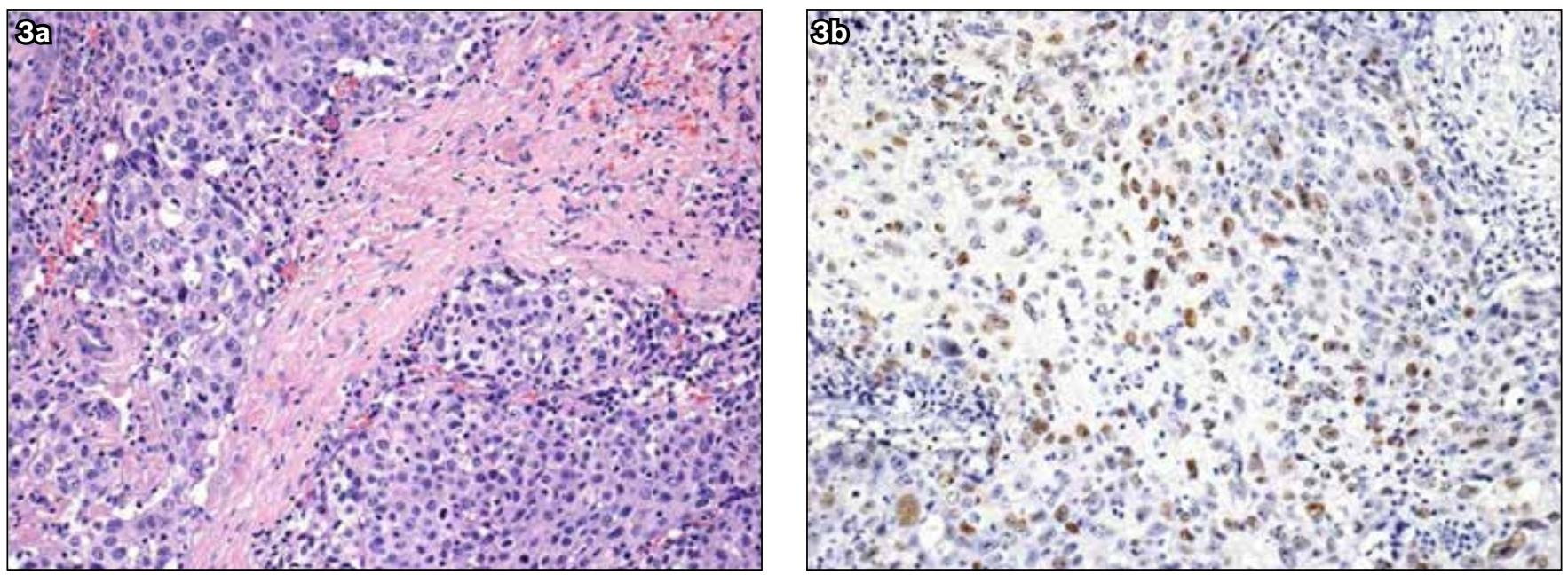

Fig. 3 (a) Photomicrographs of tumour cells show (a) differentiated non-keratinising carcinoma (Haematoxylin \& eosin, $\times 200$ ) and (b) positive Epstein-Barr virus-encoded RNA staining in tumour cells on in situ hybridisation ( $\times 200)$.

Brain metastases have been reported to occur in $20 \%-45 \%$ of patients with systemic malignancies, and autopsy reports suggest that about $10 \%$ of patients with systemic malignancies have dural metastases. ${ }^{(7)}$ The most common primary sites of dural metastases are the breast, prostate and lungs. ${ }^{(8)}$ Nevertheless, dural metastasis from NPC is exceedingly rare.(7)

The diagnostic challenge presented by dural metastasis of NPC is not only associated with the rarity of dural metastasis from NPC but also because dural metastases can be mistaken for a benign entity both clinically and radiographically. Clinically, about 20\% of dural metastases are symptomatically silent and discovered incidentally on radiological workup. ${ }^{(7)}$ Radiographically, although $M R$ imaging is useful in differentiating malignant soft-tissue tumours from benign ones, dural metastases can be mistaken for benign meningiomas that typically have a ragged border, ${ }^{(9)}$ due to identical dural tail signs on MR imaging. ${ }^{(4)}$ As disease progression is rapid, delayed diagnosis of dural metastasis of NPC also means that it might not be possible to perform appropriate surgical intervention in these patients in a timely manner.

With the dural tail sign on follow-up MR imaging as the only clinical indicator, dural metastasis from NPC was not identified early in our patient, causing an increase in intracranial pressure and subsequent neurological complications. Although the missed diagnosis may have led to fatal consequences, tragedy was narrowly avoided in our patient through prompt treatment (i.e. craniectomy and tumour resection).
In conclusion, this report highlights the diagnostic and assessment difficulties clinicians face when dealing with patients diagnosed with NPC following treatment. Clinicians need to maintain a high index of suspicion for dural metastasis in these patients when evidence such as the dural tail sign on repeat MR imaging is present. We recommend that a diagnosis of benign dural lesion be made only if a malignant lesion is excluded.

\section{REFERENCES}

1. Kuo $\mathrm{CL}$, Wang $\mathrm{MC}$, $\mathrm{Chu} \mathrm{CH}$, Shiao AS. New therapeutic strategy for treating otitis media with effusion in postirradiated nasopharyngeal carcinoma patients. J Chin Med Assoc 2012; 75:329-34.

2. Ho SY, Wang YJ, Huang PC, et al. Evaluation of the associations between the single nucleotide polymorphisms of the promoter region of the tumor necrosis factor-alpha gene and nasopharyngeal carcinoma. J Chin Med Assoc 2006; 69:351-7.

3. Ahmad A, Stefani S. Distant metastases of nasopharyngeal carcinoma: a study of 256 male patients. J Surg Oncol 1986; 33:194-7.

4. Tagle P, Villanueva P, Torrealba G, Huete I. Intracranial metastasis or meningioma? An uncommon clinical diagnostic dilemma. Surg Neurol 2002; 58:241-5.

5. Lu H, Peng L, Yuan X, et al. Concurrent chemoradiotherapy in locally advanced nasopharyngeal carcinoma: a treatment paradigm also applicable to patients in Southeast Asia. Cancer Treat Rev 2009; 35:345-53.

6. Westphal M, Heese O, de Wit M. Intracranial metastases: therapeutic options. Ann Oncol 2003; 14 Suppl 3:iii4-10.

7. Laigle-Donadey F, Taillibert S, Mokhtari K, Hildebrand J, Delattre JY. Dural metastases. J Neurooncol 2005; 75:57-61.

8. Nayak L, Abrey LE, Iwamoto FM. Intracranial dural metastases. Cancer 2009; 115:1947-53.

9. Nakamura M, Roser F, Mirzai S, et al. Meningiomas of the internal auditory canal. Neurosurgery 2004; 55:119-27; discussion 127-8. 